\title{
Code-Rate-Optimized Differentially Modulated Near-Capacity Cooperation
}

\author{
Li Wang, Member, IEEE, Lingkun Kong, Soon Xin Ng, Senior Member, IEEE, and Lajos Hanzo
}

\begin{abstract}
It is widely recognized that half-duplex-relay-aided differential decode-and-forward (DDF) cooperative transmission schemes are capable of achieving a cooperative diversity gain, while circumventing the potentially excessive-complexity and yet inaccurate channel estimation, especially in mobile environments. However, when a cooperative wireless communication system is designed to approach the maximum achievable spectral efficiency by taking the cooperation-induced multiplexing loss into account, it is not obvious whether or not the relay-aided system becomes superior to its direct-transmission based counterpart, especially, when advanced channel coding techniques are employed. Furthermore, the optimization of the transmit-interval durations required by the source and relay is an open issue, which has not been well understood in the context of half-duplex relaying schemes. Hence, we first find the optimum transmission duration, which is proportional to the adaptive channel-code rate of the source and relay in the context of Code-RateOptimized (CRO) TDMA-based DDF-aided half-duplex systems for the sake of maximizing the achievable network throughput. Then, we investigate the benefits of introducing cooperative mechanisms into wireless networks, which may be approached in the context of the proposed CRO cooperative system both from a pure capacity perspective and from the practical perspective of approaching the Discrete-input Continuous-output Memoryless Channel (DCMC) capacity with the aid of the proposed Irregular Distributed Differential (IrDD) coding aided scheme. In order to achieve a near-capacity performance at a low-complexity, an adaptive-window-duration based Multiple-Symbol Differential Sphere Detection (MSDSD) scheme is employed in the iterative detection aided receiver. Specifically, upon using the proposed near-capacity system design, the IrDD coding scheme devised becomes capable of performing within about 1.8 dB from the corresponding single-relay-aided DDF cooperative system's DCMC capacity.
\end{abstract}

Index Terms-Non-coherent communication, differential encoding, near-capacity wireless communication, adaptive-rate channel coding, avoiding channel estimation, cooperative communications, relaying, coded cooperation.

\section{BACKGROUND AND MOTIVATION}

$\mathbf{T}$ HE technological advances in integrated circuits and radio-frequency electronics facilitate the employment of ever more sophisticated signal processing and coding algorithms. At the same time, it is increasingly important to find energy- and bandwidth-efficient solutions for reliable digital

Paper approved by E. Perrins, the Editor for Modulation Theory of the IEEE Communications Society. Manuscript received March 3, 2010; revised September 2, 2010 and January 21, 2011

The research leading to these results has received funding from the European Union's Seventh Framework Programme ([FP7/2007-2013]) under grant agreement no [214625]. The financial support of the RC UK under the auspices of the UK-India Advanced Technology Centre of Wireless Communications and of the China-UK Science Bridge in $4 \mathrm{G}$ wireless communications, as well as that of the EU's Optimix project, is also gratefully acknowledged.

The authors are with the school of ECS, University of Southampton, Southampton, SO17 1BJ, UK (e-mail: \{lw5, 1k06r, sxn, 1h\}@ecs.soton.ac.uk).

Digital Object Identifier 10.1109/TCOMM.2011.061511.100115 communication over time-varying wireless channels. Multiple antenna aided diversity techniques [1] constitute powerful arrangements of mitigating the deleterious effects of fading, hence improving the end-to-end system performance, which is usually achieved by multiple co-located antenna elements at the transmitter and/or receiver. However, it is often impractical for the mobile to employ a large number of antennas due to its size- and cost- constraints. Fortunately, in multi-user wireless systems mobile stations (MSs) may cooperatively share their antennas in order to achieve uplink transmit diversity by forming a virtual antenna array (VAA) in a distributed fashion [2], [3]. On the other hand, channel estimation for a VAA-aided system may impose both an excessive complexity and a high pilot overhead, especially in mobile environments associated with relatively rapidly fluctuating channel conditions. Hence, differentially encoded transmissions combined with non-coherent detection and thus requiring no channel state information (CSI) at the receiver becomes an attractive design alternative, leading to differential modulation assisted cooperative communications [4]-[8]. Furthermore, in order to mitigate the detrimental impact of the time-selective channel on differentially encoded transmissions induced by the relative mobility amongst transceivers, multiple-symbol differential sphere detection (MSDSD) has been proposed in [9] and has been further developed for a differentially modulated cooperative system in [6], under the assumption that the channel statistics and the respective distances are known by the cooperating MSs and the base station (BS).

Naturally, in the absence of channel coding, the cooperative system performance is expected to be better than that of the direct transmission [6], [7], owing to the cooperative diversity gains and the path loss reduction. In order to further enhance the achievable cooperative performance, beneficial power allocation and relay station (RS) selection schemes [2], [7], [8], [10] have been invoked. However, they have mainly been investigated in the context of fixed and predetermined time resource allocation (TRA) between the source and RS in time-division multiple access (TDMA) scenarios. Thus, little attention has been devoted to the optimization of TRA.

Moreover, the cooperative diversity gains promised by the cooperative system considered are actually achieved at the cost of suffering a significant so-called multiplexing loss ${ }^{1}$ compared to direct transmissions, which is imposed by the half-duplex communications of practical transceivers. Further-

${ }^{1}$ In fact, in pursuit of recovering the multiplexing loss suffered by the half-duplex-relay aided system without compromising the attainable transmit diversity gains, a so-called successive relaying scheme has been recently proposed in [11], which may be used in the context of our differentially encoded transmission system as well. This suggestion of the anonymous reviewer is gratefully acknowledged. 
more, the cooperative diversity gains achieved by the relayaided system over its direct-transmission based counterpart may become modest in practical channel coded scenarios, where the interleaving and channel coding gains dominate. Therefore, when a cooperative wireless communication system is designed to approach the maximum achievable spectral efficiency by taking the cooperation-induced multiplexing loss into account, it is not obvious, whether or not the repetition-based relay-aided system becomes superior to its direct-transmission based counterpart. This may be achieved by investigating the noncoherent Discrete-input Continuousoutput Memoryless Channel (DCMC) capacity [12] of the cooperative network in comparison to that of the directtransmission based system.

Against this background, the novel contributions of this treatise are as follows: 1). We deduce the optimum TRA policy for the sake of maximizing the differetial decodeand-forward (DDF) aided cooperative system's capacity by utilizing information theoretical tools. It becomes useful in the ensuing design of near-capacity coding/decoding schemes conceived for cooperative systems, since the channel code rate employed by the source and RS is directly related to their allocated transmission duration, and may be adaptively controlled according to the proposed TRA scheme. 2). We answer the fundamental dilemma, whether it is worth incorporating cooperative mechanisms into wireless networks. 3). Inspired by the philosophy of distributed turbo codes [13] proposed for “distributed MIMO" systems, a novel Irregular Distributed Differential (IrDD) coding scheme is conceived for the DDFaided cooperative system, in order to maximize the system's spectral efficiency. 4). Based on our low-complexity nearcapacity design criterion, we propose a practical framework for designing an IrDD-assisted cooperative system, which is capable of performing close to the system's non-coherent DCMC capacity. 5). In order to further reduce the complexity imposed, while maintaining a near-capacity performance, the so-called adaptive-window-duration based iterative MSDSD scheme is proposed.

The rest of this paper is organized as follows. In Section II, a brief description of the single-relay-aided DDF cooperative system architecture and the channel model considered are presented. Section III reviews the non-coherent DCMC channel capacity of differentially modulated direct transmissions, followed by the investigation of the CRO single-relay-aided DDF cooperative network's DCMC capacity in Section IV. Then, in Section $\mathrm{V}$ a cooperative transceiver is designed, which is capable of approaching the non-coherent network's DCMC capacity using our low-complexity near-capacity system design procedure conceived in Section VI. Our simulation results and discussions are provided in Section VII. Finally, we conclude in Section VIII.

\section{System Architecture \& Channel Model}

The TDMA-based DDF-aided cooperative cellular uplink [7] is considered without any loss of generality, where no CSI estimation is required. For the sake of simplicity, we consider the single-relay-assisted scenario of Fig. 1, where only one cooperating MS is activated in order to decode and re-encode the signal received from the source MS prior to forwarding

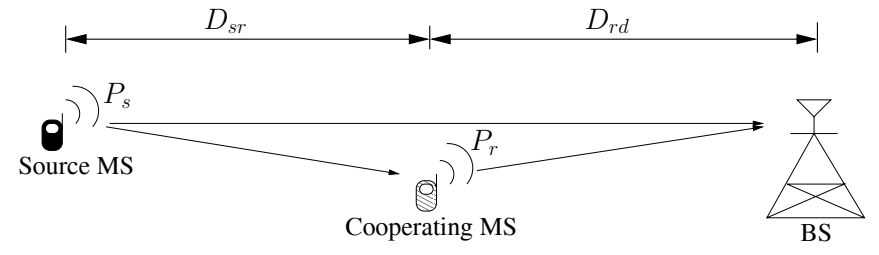

Fig. 1. Single-relay-aided cooperative cellular uplink.

the signal to the BS. The employment of a single antenna for each MS is assumed, owing to the cost- and size-constraints of MSs. In order to avoid channel estimation in the cooperative system, both the source and relay MSs employ conventional differential modulation schemes, e.g. DQPSK.

For the sake of carrying out a fair comparison between the cooperative system and the direct transmission system as well as to put our emphasis on investigating the maximum achievable transmission rate of a general repetition-based cooperative scenario, we stipulate the simplifying assumption of equal power allocation $\left(P_{s}=P_{r}=P\right)$ and mid-point RS location $\left(D_{s r}=D_{r d}\right)$, as shown in Fig. 1 . Note that $P$ is the transmit power employed by the classic direct transmission system, while $P_{s}$ and $P_{r}$ are the power used by the source and relay, respectively, when they are transmitting. However, note that the investigations carried out in this treatise may also be readily applied to other power allocation and relay location arrangements, such as for example, the partial compensation based power control strategy proposed in [14], which is capable of reducing the transmit power, hence extending the battery recharge period and thus reducing the health risks. For analytical simplicity, we assume $D_{s r}+D_{r d}=D_{s d}$. Moreover, the normalized average power $\sigma_{i, j}^{2}$ at the output of the channel is inversely proportional to the inter-node distance $D_{i, j}$, which may be rewritten as

$$
\sigma_{i, j}^{2}=D_{i, j}^{-v}, i, j \in\{s, r, d\},
$$

where $v$ denotes the path-loss exponent.

In order to provide a good approximation for TDMAbased cooperative systems and to facilitate the study of the non-coherent detection-based channel capacity, we consider a time-selective block-fading Rayleigh channel [15], where the fading coefficients are assumed to change in an independent and identically-distributed (i.i.d.) manner from block to block, while exhibiting correlation within a transmission block according to the Doppler frequency governed by the relative movement of the tranceivers.

In the scenario of the single-relay-aided transmission of Fig. 1 over a time-selective block-fading channel, the $T_{b}$ signals consecutively received within one of the $\frac{L_{s}}{T_{b}}$ fading blocks at the RS during the broadcast Phase I, where $L_{s}$ is the total number of symbols transmitted from the source MS during Phase I, may be formulated as:

$$
\mathbf{y}_{r}^{I}=\sqrt{P_{s}} \mathbf{S}_{D, s}^{I} \mathbf{h}_{s r}+\mathbf{w}_{r}^{I},
$$

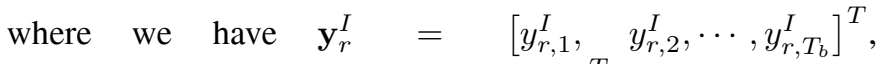
$\mathbf{h}_{s r}=\left[h_{s r, 1}, \quad h_{s r, 2}, \cdots, h_{s r, T_{b}}\right]^{T}, \quad$ and $\quad \mathbf{w}_{r}^{I}=$ $\left[w_{r, 1}^{I}, \quad w_{r, 2}^{I}, \cdots, w_{r, T_{b}}^{I}\right]^{T}$ representing the received signal column vector, the channel impulse response (CIR) column 
vector and the Gaussian noise column vector having a distribution of $\mathcal{C N}\left(0,2 \sigma_{w}^{2} \mathbf{I}_{T_{b}}\right)$, respectively. Note that $\mathbf{I}_{T_{b}}$ denotes a $\left(T_{b} \times T_{b}\right)$ identity matrix. The diagonal matrix $\mathbf{S}_{D, s}^{I}$ in (2) hosts the $T_{b}$ consecutively transmitted signals within a fading block during Phase I, which may be expressed as $\mathbf{S}_{D, s}^{I}=\operatorname{diag}\left\{\mathbf{s}_{\mathrm{s}}^{\mathrm{I}}\right\}$, where we have $\mathbf{s}_{s}^{I}=\left[s_{s, 1}^{I}, \quad s_{s, 2}^{I}, \cdots, s_{s, T_{b}}^{I}\right]^{T}$. Similarly, the signals consecutively received within one of the $\frac{L_{s}}{T_{b}}$ fading blocks at the BS from the source MS during Phase I can be expressed as:

$$
\mathbf{y}_{d}^{I}=\sqrt{P_{s}} \mathbf{S}_{D, s}^{I} \mathbf{h}_{s d}+\mathbf{w}_{d}^{I},
$$

and the signals consecutively arriving at the BS within one of the $\frac{L_{r}}{T_{b}}$ fading blocks during the relaying Phase II is formulated as:

$$
\mathbf{y}_{d}^{I I}=\sqrt{P_{r}} \mathbf{S}_{D, r}^{I I} \mathbf{h}_{r d}+\mathbf{w}_{d}^{I I} .
$$

Note that $L_{r}$ is the total number of symbols transmitted from the RS when the source MS keeps silent. In (3) and (4), $\mathbf{y}_{d}^{I}$ and $\mathbf{y}_{d}^{I I}$ are both $T_{b}$-element received signal vectors. Furthermore, $\mathbf{h}_{s d} \in \mathbb{C}^{T_{b} \times 1}$ and $\mathbf{h}_{r d} \in \mathbb{C}^{T_{b} \times 1}$ represent CIR column vectors, while the Gaussian noise column vectors $\mathbf{w}_{d}^{I}, \mathbf{w}_{d}^{I I} \in \mathbb{C}^{T_{b} \times 1}$ have a distribution of $\mathcal{C N}\left(0,2 \sigma_{w}^{2} \mathbf{I}_{T_{b}}\right)$. Moreover, the diagonal matrix $\mathbf{S}_{D, r}^{I I}=\operatorname{diag}\left\{\mathbf{s}_{r}^{I I}\right\}$, where we have $\mathbf{s}_{r}^{I}=\left[s_{r, 1}^{I I}, s_{r, 2}^{I I}, \cdots, s_{r, T_{b}}^{I I}\right]^{T}$, hosts the $T_{b}$ signals consecutively transmitted from the RS within a fading block during Phase II.

Note that given the assumption of $T_{b}$-length Rayleigh block fading, $\mathbf{h}_{i, j}, i, j \in\{s, r, d\}$ in (2), (3) or (4) obeys a complex-valued Gaussian distribution of $\mathcal{C N}\left(0, \Sigma_{h_{i j}}\right)$, where the $\left(T_{b} \times T_{b}\right)$-element covariance matrix $\Sigma_{h_{i j}}=\mathcal{E}\left\{\mathbf{h}_{i j} \mathbf{h}_{i j}^{H}\right\}$ may be expressed as:

$$
\Sigma_{h_{i j}}=\left[\begin{array}{cccc}
\varphi_{i j}^{t}[0] & \varphi_{i j}^{t}[1] & \cdots & \varphi_{i j}^{t}\left[T_{b}-1\right] \\
\varphi_{i j}^{t}[-1] & \varphi_{i j}^{t}[0] & \cdots & \varphi_{i j}^{t}\left[T_{b}-2\right] \\
\vdots & \vdots & \ddots & \vdots \\
\varphi_{i j}^{t}\left[1-T_{b}\right] & \varphi_{i j}^{t}\left[2-T_{b}\right] & \cdots & \varphi_{i j}^{t}[0]
\end{array}\right],
$$

where $i, j \in\{s, r, d\}$ and $\varphi_{i j}^{t}[\kappa]$ represents the channel's autocorrelation function, which can be expressed as $\varphi_{i j}^{t}[\kappa] \triangleq$ $\mathcal{E}\left\{h_{i j,(n+\kappa)} h_{i j, n}^{*}\right\}=\sigma_{i j}^{2} \cdot J_{0}\left(2 \pi f_{d, i j} \kappa\right)$, with $J_{0}(\cdot)$ denoting the zeroth-order Bessel function of the first kind and $f_{d, i j}$ representing the normalized Doppler frequency. For the sake of simplicity, $f_{d, i j}=f_{d}, i, j \in\{s, r, d\}$ is assumed for each link.

\section{NON-COHERENT CHANNEL CAPACITY FOR POINT-TO-POINT LINKS}

Let us first focus our attention on the non-coherent DCMC capacity of each link of the DDF-aided cooperative system seen in Fig. 1, based on which the non-coherent DCMC network capacity of the cooperative system will be studied in Section IV. Without loss of generality, we may arrive at a generalized point-to-point transmission model by omitting the subscripts and superscripts in (2), (3) or (4), yielding:

$$
\mathbf{y}=\sqrt{P} \mathbf{S}_{D} \mathbf{h}+\mathbf{w} .
$$

The probability density function (PDF) of the received signal vector $\mathbf{y}$ in (6) is conditioned on the transmitted signal vector s, which may be readily expressed as [16]:

$$
p(\mathbf{y} \mid \mathbf{s})=\frac{\exp \left(-\mathbf{y}^{H} \Psi^{-1} \mathbf{y}\right)}{\operatorname{det}(\pi \Psi)},
$$

where we have $\Psi=\mathcal{E}\left\{\mathbf{y} \mathbf{y}^{H} \mid \mathbf{s}\right\}=\mathbf{S}_{d} P \mathcal{E}\left\{\mathbf{h} \mathbf{h}^{H}\right\} \mathbf{S}_{d}^{H}+2 \sigma_{w}^{2} \mathbf{I}_{T_{b}}$. Since each element of the $\left(T_{b} \times 1\right)$-component differentially transmitted signal vector $\mathbf{s}$ is chosen independently from an $M_{c}$-point constellation set $\mathcal{M}_{c}$ with equal probabilities, the non-coherent DCMC capacity can be expressed as a function of the SNR $\gamma$ as follows:

$$
C_{p 2 p}(\gamma)=\frac{1}{T_{b}} I(\mathbf{s} ; \mathbf{y})=\frac{1}{T_{b}}(H(\mathbf{y})-H(\mathbf{y} \mid \mathbf{s})),
$$

where $I(\mathbf{a} ; \mathbf{b})$ represents the average mutual information (MI) per symbol between the channel input a and the corresponding channel output $\mathbf{b}$, while $H(\mathbf{x})$ represents the differential entropy [17] of a random vector x. According to [17], $H(\mathbf{y} \mid \mathbf{s})$ may be readily calculated as:

$$
H(\mathbf{y} \mid \mathbf{s})=\frac{1}{M_{c}^{T_{b}}} \sum_{\breve{\mathbf{s}} \in \chi} \log \operatorname{det}(\pi \mathrm{e} \Psi) \text { bits. }
$$

On the other hand, the entropy $H(\mathbf{y})$ of the continuous-valued faded and noise-contaminated received signal vector $\mathbf{y}$ cannot be evaluated in a closed form. When the fading block size $T_{b}$ over which the fading envelope is assumed to be correlated is limited, a practical approach to the numerical evaluation of $H(\mathbf{y})$ is to carry out Monte-Carlo integration as follows [18]:

$$
\begin{aligned}
H(\mathbf{y}) & =-\int p(\mathbf{y}) \log p(\mathbf{y}) d \mathbf{y} \\
& =-\mathcal{E}\left\{\log _{2}\left(\frac{1}{M_{c}^{T_{b}} \operatorname{det}(\pi \Psi)} \sum_{\breve{\mathbf{s}} \in \chi} \exp \left(-\mathbf{y}^{H} \Psi^{-1} \mathbf{y}\right)\right)\right\},
\end{aligned}
$$

where $\chi$ is the set of all $M_{c}^{T_{b}}$ hypothetically transmitted

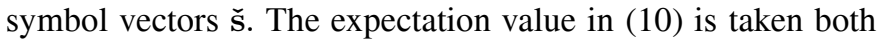
with respect to different CIR realizations and to the noise.

The non-coherent DCMC capacity of the time-selective block-fading channel computed from (8)-(10) using the DQPSK modulation scheme is plotted in Fig. 2 for various fading block sizes of $T_{b}=2,4$ and 7 as well as for various normalized Doppler frequencies of $f_{d}=0,0.01$ and 0.03. As observed in Fig. 2, although an identical differential modulation scheme is employed, the maximum achievable spectral efficiency is dependent on both the fading block size $T_{b}$ and the fading correlation over blocks characterized by the corresponding covariance matrix $\mathcal{E}\left\{\mathbf{h} \mathbf{h}^{H}\right\}$.

\section{The Code-RAte-Optimization For CoOperative NETWORK}

\section{A. DCMC Capacity for the DDF-Aided Cooperative Network}

Based on the general upper and lower bounds on the capacity of half-duplex relay systems presented in [10] and on the fact that in our DDF-aided half-duplex relay system, the source MS remains silent during Phase II, we may obtain 


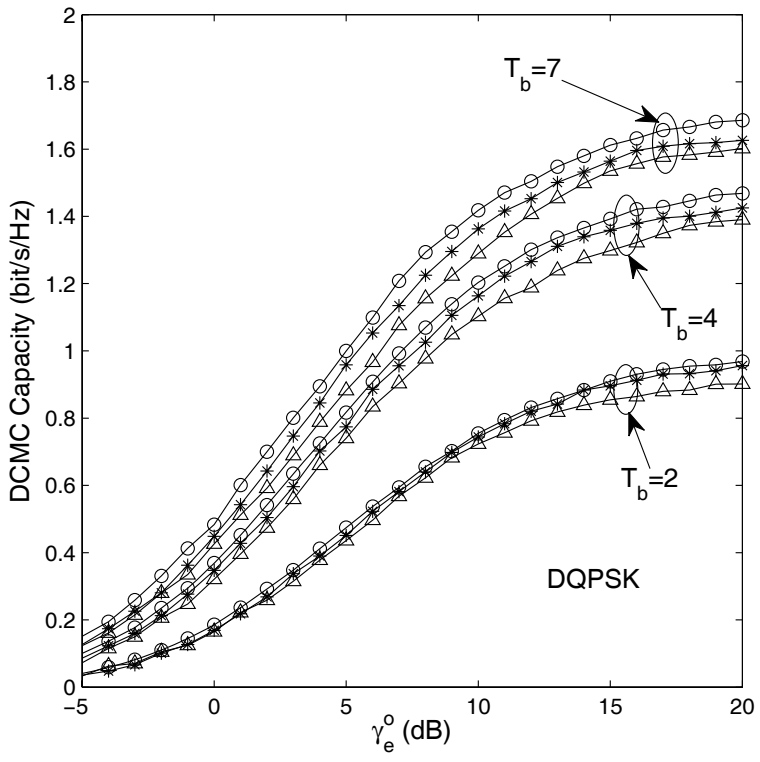

Fig. 2. Non-coherent DCMC capacity of the single-input single-output timeselective block-fading channel. 1). Circle-decorated lines: $f_{d}=0 ; 2$ ). Stardecorated lines: $\left.f_{d}=0.01 ; 3\right)$. Triangle-decorated lines: $f_{d}=0.03$.

simplified capacity upper and lower bounds for the DDF system of Fig. 1 as:

$$
\begin{aligned}
C_{\text {coop }}\left(\gamma_{e}^{o}, \alpha\right) \leq \min \left\{\alpha I\left(\mathbf{s}_{s}^{I} ; \mathbf{y}_{d}^{I}, \mathbf{y}_{r}^{I}\right),\right. \\
\left.\alpha I\left(\mathbf{s}_{s}^{I} ; \mathbf{y}_{d}^{I}\right)+(1-\alpha) I\left(\mathbf{s}_{r}^{I I} ; \mathbf{y}_{d}^{I I}\right)\right\},
\end{aligned}
$$

and

$$
\begin{aligned}
& C_{\text {coop }}\left(\gamma_{e}^{o}, \alpha\right) \geq \min \left\{\alpha I\left(\mathbf{s}_{s}^{I} ; \mathbf{y}_{r}^{I}\right),\right. \\
&\left.\alpha I\left(\mathbf{s}_{s}^{I} ; \mathbf{y}_{d}^{I}\right)+(1-\alpha) I\left(\mathbf{s}_{r}^{I I} ; \mathbf{y}_{d}^{I I}\right)\right\},
\end{aligned}
$$

where the TRA-factor $\alpha$ is defined as:

$$
\alpha \triangleq \frac{L_{s}}{L_{s}+L_{r}}=\frac{R_{r}}{R_{s}+R_{r}},
$$

since the ratio of the time durations $\left(L_{s}, L_{r}\right)$ used by the source and $\mathrm{RS}$ is inversely proportional to the ratio of the channel code rates $\left(R_{s}, R_{r}\right)$ employed by them. The capacity upper and lower bounds of (12) and (13) are the functions of the network's overall equivalent SNR, i.e. of $\gamma_{e}^{o}$ and the TRA-factor $\alpha$ of (14). Note that the terminology of 'equivalent SNR' is used here to indicate the fact that it quantifies the ratio of the transmit power and the receiver's noise, which are measured at physically different points. This is in line with [19] for example. $\gamma_{e}^{o}=\frac{P}{2 \sigma_{w}^{2}}$ represents the network's overall equivalent SNR, which is equal to the sum of the the equivalent SNRs, $\gamma_{e}^{s}=\frac{P_{s}}{2 \sigma_{w}^{2}}$ and $\gamma_{e}^{r}=\frac{P_{r}}{2 \sigma_{w}^{2}}$ at the source and relay transmitters.

On the other hand, given a fading block size $T_{b}$ and a $M_{c^{-}}$ ary DPSK scheme, the actual transmission rate, $R_{\text {coop }}$, of the cooperative system of Fig. 1 is a function of both $R_{s}$ and $\alpha$, which may be expressed as:

$$
R_{\text {coop }}\left(R_{s}, \alpha\right)=\alpha R_{s} \frac{T_{b}-1}{T_{b}} \log _{2} M_{c} .
$$

Furthermore, in order for the RS to decode the received signal correctly thus avoiding the potential error propagation, the source transmission rate should be below the non-coherent constrained information rate of the source-relay (SR) link, thus we have:

$$
R_{\text {coop }}\left(R_{s}, \alpha\right) \leq \alpha C_{s r}\left(\gamma_{e}^{o}\right)=\alpha I\left(\mathbf{s}_{s}^{I} ; \mathbf{y}_{r}^{I}\right)
$$

Consequently, according to (12), (13) and (16), the errorfree-relaying-based DDF cooperative network capacity can be written as:

$$
\begin{aligned}
C_{\text {coop }}^{\mathrm{DDF}}\left(\gamma_{e}^{o}, \alpha\right)=\min & \left\{\alpha I\left(\mathbf{s}_{s}^{I} ; \mathbf{y}_{r}^{I}\right)\right. \\
& \left.\alpha I\left(\mathbf{s}_{s}^{I} ; \mathbf{y}_{d}^{I}\right)+(1-\alpha) I\left(\mathbf{s}_{r}^{I I} ; \mathbf{y}_{d}^{I I}\right)\right\} .
\end{aligned}
$$

where the constrained information rates of $I\left(\mathbf{s}_{s}^{I} ; \mathbf{y}_{d}^{I}\right)$, $I\left(\mathbf{s}_{r}^{I I} ; \mathbf{y}_{d}^{I I}\right)$ and $I\left(\mathbf{s}_{s}^{I} ; \mathbf{y}_{r}^{I}\right)$ can be evaluated based on (8)-(10). Hence, given the target transmission rate $R_{\text {coop }}\left(R_{s}, \alpha\right)$ of (15), the question arises as to how to design the cooperative system of Fig. 1 by optimizing the TRA or equivalently optimizing the code rate allocation between the source and RS in the interest of requiring the globally minimum $\gamma_{e}^{o}$, which has to be smaller than the minimum $\gamma_{e}^{o}$ required by its direct-transmission based counterpart in order to make the cooperative system design meaningful. The challenging issue will be addressed in the rest of this paper.

\section{B. Optimum Code Rate for the Cooperative Network}

Lemma 1: The optimum TRA-factor $\hat{\alpha}^{\text {opt }}$, which maximizes the achievable information rate of a single-relay-aided DDFbased cooperative transmission, is given by:

$$
\hat{\alpha}^{o p t}\left(\gamma_{e}^{o}\right)=\frac{I\left(\mathbf{s}_{r}^{I I} ; \mathbf{y}_{d}^{I I}\right)}{I\left(\mathbf{s}_{s}^{I} ; \mathbf{y}_{r}^{I}\right)-I\left(\mathbf{s}_{s}^{I} ; \mathbf{y}_{d}^{I}\right)+I\left(\mathbf{s}_{r}^{I I} ; \mathbf{y}_{d}^{I I}\right)},
$$

yielding the globally maximum achievable transmission rate of

$$
C_{\text {coop }}^{\mathrm{DDF}}\left(\gamma_{e}^{o}, \hat{\alpha}^{o p t}\right)=\frac{I\left(\mathbf{s}_{s}^{I} ; \mathbf{y}_{r}^{I}\right) I\left(\mathbf{s}_{r}^{I I} ; \mathbf{y}_{d}^{I I}\right)}{I\left(\mathbf{s}_{s}^{I} ; \mathbf{y}_{r}^{I}\right)-I\left(\mathbf{s}_{s}^{I} ; \mathbf{y}_{d}^{I}\right)+I\left(\mathbf{s}_{r}^{I I} ; \mathbf{y}_{d}^{I I}\right)}
$$

The proof of Lemma 1 provided in [20] demonstrated that the first term within the min function of (17) increases with $\alpha$, while the second term decreases with $\alpha$. Thus, the optimum value of $\alpha$ is the point obtained by equating the two arguments, which gives rise to (18).

Lemma 2: In order to attain the globally highest achievable information rate of a single-relay-aided DDF-based $M_{c}$-ary DPSK modulated cooperative scheme over a time-selective block fading channel associated with a fading block size of $T_{b}$, the optimum code rate pair $\left\{\hat{R}_{s}^{\text {opt }}, \hat{R}_{r}^{\text {opt }}\right\}$ employed by the source and relay MSs is given by:

$$
\begin{gathered}
\hat{R}_{s}^{o p t}=\frac{T_{b} I\left(\mathbf{s}_{s}^{I} ; \mathbf{y}_{r}^{I}\right)}{\left(T_{b}-1\right) \log _{2} M_{c}} ; \\
\hat{R}_{r}^{o p t}=\frac{T_{b} I\left(\mathbf{s}_{s}^{I} ; \mathbf{y}_{r}^{I}\right) I\left(\mathbf{s}_{r}^{I I} ; \mathbf{y}_{d}^{I I}\right)}{\left(T_{b}-1\right)\left[I\left(\mathbf{s}_{s}^{I} ; \mathbf{y}_{r}^{I}\right)-I\left(\mathbf{s}_{s}^{I} ; \mathbf{y}_{d}^{I}\right)\right] \log _{2} M_{c}} .
\end{gathered}
$$

Proof of Lemma 2: Using the results of (18) and (19), as well as utilizing the actual network transmission rate expression of (15), we have:

$$
\hat{R}_{s}^{o p t}=\frac{T_{b} \cdot C_{c o o p}^{\mathrm{DDF}}\left(\gamma_{e}^{o}, \hat{\alpha}^{o p t}\right)}{\left(T_{b}-1\right) \cdot \hat{\alpha}^{o p t} \cdot \log _{2} M_{c}}=\frac{T_{b} I\left(\mathbf{s}_{s}^{I} ; \mathbf{y}_{r}^{I}\right)}{\left(T_{b}-1\right) \log _{2} M_{c}} .
$$




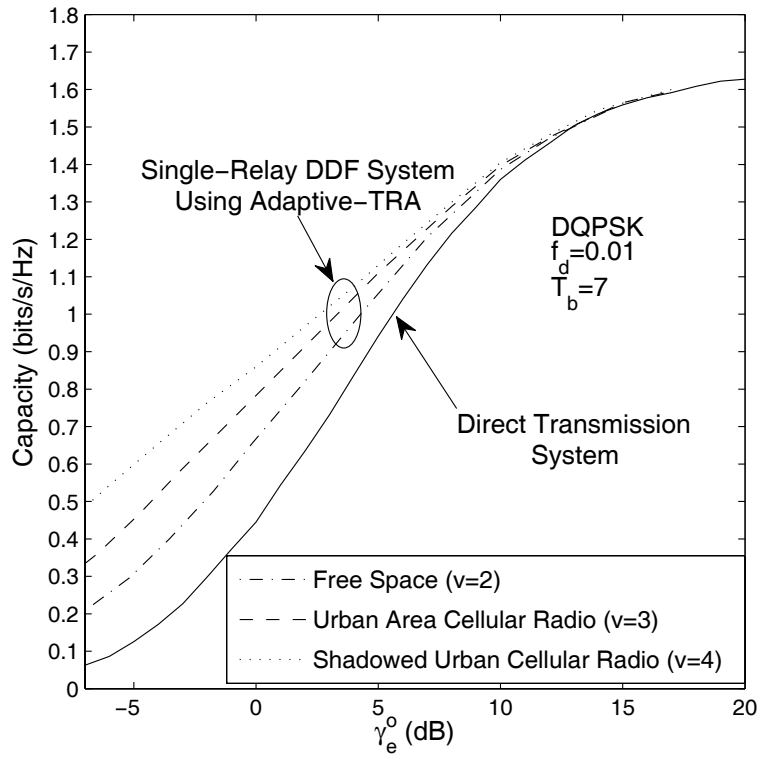

Fig. 3. Capacity comparison of the single-relay-aided cooperative system and its direct-transmission based counterpart.

Then, according to the definition of $\alpha$ in (14), the corresponding capacity-achieving code rate $\hat{R}_{r}^{o p t}$ that can be employed by the RS may be computed with the aid of (18) and (22) as follows:

$$
\begin{aligned}
\hat{R}_{r}^{o p t} & =\frac{\hat{\alpha}^{o p t} \hat{R}_{s}^{o p t}}{1-\hat{\alpha}^{o p t}} \\
& =\frac{T_{b} I\left(\mathbf{s}_{s}^{I} ; \mathbf{y}_{r}^{I}\right) I\left(\mathbf{s}_{r}^{I I} ; \mathbf{y}_{d}^{I I}\right)}{\left(T_{b}-1\right)\left[I\left(\mathbf{s}_{s}^{I} ; \mathbf{y}_{r}^{I}\right)-I\left(\mathbf{s}_{s}^{I} ; \mathbf{y}_{d}^{I}\right)\right] \log _{2} M_{c}} .
\end{aligned}
$$

This completes the proof of Lemma 2 .

\section{Capacity Comparison and Discussions}

In order to gain an insight into the benefits of the singlerelay-assisted DDF cooperative system over its conventional direct-transmission based counterpart from a pure capacity perspective, the DCMC capacity of the CRO cooperative system is depicted in comparison to that of the direct-transmission based one in Fig. 3. It may be observed in the figure that if the overall equivalent SNR is relatively low, the CRO DDF cooperative system exhibits a significantly higher capacity than its direct-transmission based counterpart in typical urban cellular scenarios. More specifically, in a shadowed urban area associated with $v=4$, the DDF system only requires one sixth of the total transmit power necessitated by its directtransmission based counterpart in order to achieve a spectral efficiency of $0.5 \mathrm{bits} / \mathrm{s} / \mathrm{Hz}$. However, the achievable capacity gain may be substantially reduced, if we encounter a freespace propagation scenario associated with $v=2$, owing to the reduced path-loss-related power-gain achieved in the cooperative system. Moreover, as the overall equivalent SNR increases to a relatively high value, the benefits of invoking a single-relay-aided cooperative system for achieving a high spectral efficiency erode.

\section{Transceiver Design for the DDF-Aided COOPERATIVE SYSTEM}

\section{A. Capacity-Achieving Multiple-Symbol Differential Sphere Detection}

1) Soft-Input Soft-Output MSDSD: Let us now briefly review the Soft-Input Soft-Output (SISO) MSDSD scheme, which will be shown to be a capacity-achieving differential detector in Section V-A2 and thus will be used afterwards in our transceiver design for the DDF-aided cooperative system. In light of the conditional PDF $p(\mathbf{y} \mid \mathbf{s})$ of (7) derived for the non-coherently received signal vector $y$ in (6), the $a$ posteriori Log-Likelihood-Ratio (LLR) of the $k$ th transmitted bit $x_{k}$ at the output of the maximum-a-posterori multiplesymbol differential detector (MAP-MSDD) [9], which jointly and differentially detects $\left(N_{\text {wind }}-1\right)$ data symbols, can be evaluated with the aid of 'max-sum' approximation as [21]:

$$
\begin{aligned}
& L_{D}\left(x_{k}\right)=\ln \frac{\sum_{\mathbf{x} \in \mathbb{X}_{k,+1}} \exp \left[-\mathbf{y}^{H} \Psi(\mathbf{x}) \mathbf{y}+\ln (\operatorname{Pr}(\mathbf{x}))\right]}{\sum_{\mathbf{x} \in \mathbb{X}_{k,-1}} \exp \left[-\mathbf{y}^{H} \Psi(\mathbf{x}) \mathbf{y}+\ln (\operatorname{Pr}(\mathbf{x}))\right]} \\
& \approx-\left\|\mathbf{U} \hat{\mathbf{s}}_{M A P}^{x_{k}=+1}\right\|^{2}+\ln \left[\operatorname{Pr}\left(\hat{\mathbf{x}}_{M A P}^{x_{k}=+1}\right)\right] \\
& +\left\|\mathbf{U} \hat{\mathbf{s}}_{M A P}^{x_{k}=-1}\right\|^{2}-\ln \left[\operatorname{Pr}\left(\hat{\mathbf{x}}_{M A P}^{x_{k}=-1}\right)\right]
\end{aligned}
$$

where $\mathbb{X}_{k,+1}$ represents the set of $\frac{M_{c}^{\left(N_{\text {wind }}-1\right)}}{2}$ number of legitimate transmitted bit vectors $\mathbf{x}$ associated with $x_{k}=+1$, and similarly, $\mathbb{X}_{k,-1}$ is defined as the set corresponding to $x_{k}=-1$. Furthmore, $\operatorname{Pr}(\mathbf{x})$ is the a priori probability. In (26) $\mathbf{U}$ is an upper-triangular matrix, which can be obtained as $\mathbf{U} \triangleq(\mathbf{F} \operatorname{diag}\{\mathbf{y}\})^{*}$, with $\mathbf{F}$ also being an upper-triangular matrix generated using the Cholesky factorization of the matrix $\left(\Sigma_{h}+2 \sigma_{w}^{2} \mathbf{I}_{N_{\text {wind }}}\right)^{-1}$. Consequently, thanks to the uppertriangular structure of the matrix $\mathbf{U}, \hat{\mathbf{s}}_{M A P}^{x_{k}=b}$ and $\hat{\mathbf{x}}_{M A P}^{x_{k}=b}$ of (26), which represent the MAP-algorithm-based symbol vector estimate and the MAP-based bit vector estimate, respectively, may be obtained with the aid of the low-complexity sphere detection (SD) algorithm of [21] by fixing the $k$ th bit value to $b,(b=-1$ or +1$)$.

In order to visualize the EXtrinsic Information Transfer (EXIT) charateristics [22] of the SISO MSDSD scheme, in Fig. 4 we plot the EXIT curves associated with different observation window sizes of $N_{\text {wind }}$ for the MSDSD, which is assumed to be employed by the RS in the scenario of the DDFaided cooperative system of Fig. 1. Under the assumption of a path loss exponent of $v=3$ and the employment of DQPSK, the resultant EXIT curves drawn in dashed lines in Fig. 4 are obtained by evaluating the extrinsic MI, $I_{E}$, at the output of the MSDSD for a given input stream of bit LLRs along with the a priori MI $I_{A}$ at $\gamma_{e}^{o}=-2.3 \mathrm{~dB}$. As indicated by the increasingly higher dashed EXIT curve in Fig. 4, significantly enhanced iterative gains may be achieved in comparison to the conventional differential detection (CDD) assisted system using $N_{\text {wind }}=2$, when jointly and differentially detecting an increased number of data symbols using the MSDSD in conjunction with $N_{\text {wind }}>2$.

2) Maximum Achievable Rate Versus the Capacity - An EXIT Chart Perspective: According to the area properties of EXIT charts [22], the area $\mathcal{A}$ under the bit-based EXIT curve of a soft-detector/soft-demapper is approximately equal 


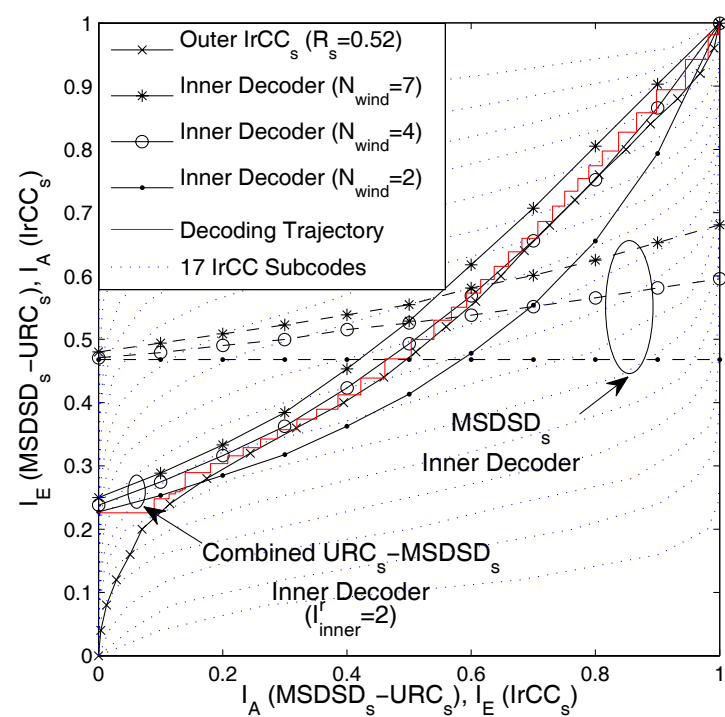

Fig. 4. EXIT curves of the MSDSD for various values of $N_{\text {wind }}$ (DQPSK, $\left.\gamma_{e}^{o}=-2.3 \mathrm{~dB}, v=3, f_{d}=0.01\right)$.

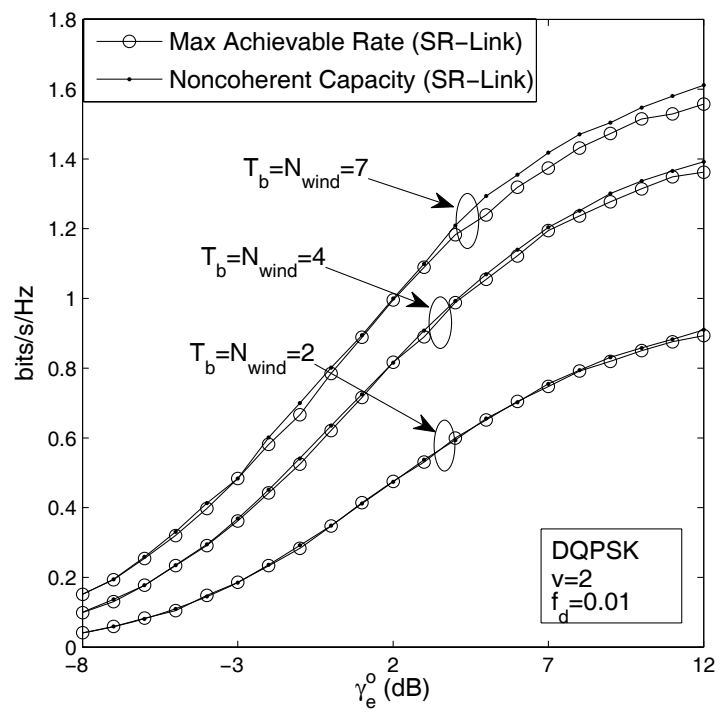

Fig. 5. Maximum achievable rate of the single-input single-output differentially encoded QPSK modulated system using the MSDSD in the timeselective block-fading channel associated with different block length $T_{b}$.

to the maximum possible code rate of the outer channel code that can be employed to achieve near-error-free transmissions. Hence, the maximum achievable near-error-free transmission rate $R_{p 2 p, \max }$ of a differentially encoded point-to-point system is computed as: $R_{p 2 p, \max }=\left(\frac{T_{b}-1}{T_{b}} \log _{2} M_{c}\right) \cdot \mathcal{A}$ bits $/ \mathrm{s} / \mathrm{Hz}$, which may be improved with the aid of the MSDSD. The ratio of $\frac{T_{b}-1}{T_{b}}$ accounts for the modest rate-loss induced by the known reference symbol of the classic differential signalling process.

In the sequel, the maximum achievable transmission rate over the SR-link employing the MSDSD may be plotted against the SNR, as shown in Fig. 5, by evaluating the area under the corresponding EXIT curve of the MSDSD. The MAP-based MSDSD employing the highest possible observation window size, namely $N_{\text {wind }}=T_{b}$, can be re-

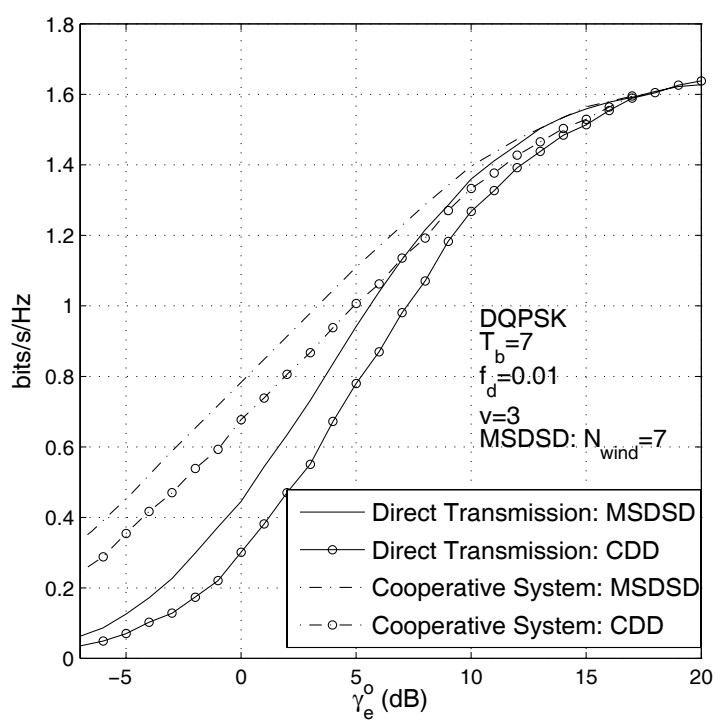

Fig. 6. Maximum achievable rate of the CDD- and MSDSD-aided systems for both direct transmission and the relay-aided transmission.

garded as the optimum differential detector in the interest of approaching the theoretically maximum transmission rate for a given differentially encoded modulation scheme. Observe in Fig. 5 that the theoretical maximum achievable rate curves of the MSDSD-aided SR-transmission portrayed for various fading block lengths $T_{b}$ almost coincide with the non-coherent DCMC capacity of the SR-link. The slight gap between them is due to the employment of the "max-sum" approximation algorithm of (26) when calculating the a posteriori LLRs.

On the other hand, in the light of (19) in Lemma 1, the theoretical maximum achievable rate of the DDF-aided cooperative system employing the CDD and the MSDSD may be calculated based on the aforementioned maximum attainable 'near-error-free' transmission rate $R_{p 2 p, \max }$ of each point-to-point link. Now the maximum achievable rate of the cooperative system and its direct-transmission based counterpart are plotted against the overall equivalent SNR $\gamma_{e}^{o}$ in Fig. 6 for both the CDD- and MSDSD-aided scenarios. Observe in Fig. 6 that within the SNR range of interest, an approximately $0.15-0.2 \mathrm{bits} / \mathrm{s} / \mathrm{Hz}$ higher 'near-error-free' transmission rate can be supported with the aid of the MSDSD in comparison to the CDD-aided scheme for the direct transmission and cooperative systems, respectively. This is because in contrast to the CDD, the MSDSD makes a decision about a block of $\left(N_{\text {wind }}-1\right)$ consecutive symbols based on $N_{\text {wind }}$ successively received symbols, hence exploiting the correlation of the fading channels. In other words, the correlation between the consecutive phase distortions imposed by the channel on the $N_{\text {wind }}$ consecutive DPSK symbols can be exploited by the MSDSD. As a result, the MSDSD is advocated in our ensuing high-spectral-efficiency cooperative system design.

\section{B. Irregular Distributed Differential Encoding/Decoding}

The transceiver architecture proposed for the single-relayaided DDF cooperative system is portrayed in Fig. 7. At the transmitter of the source MS of Fig. 7, we use a conventional differentially encoded modulation scheme, such as DQPSK, 


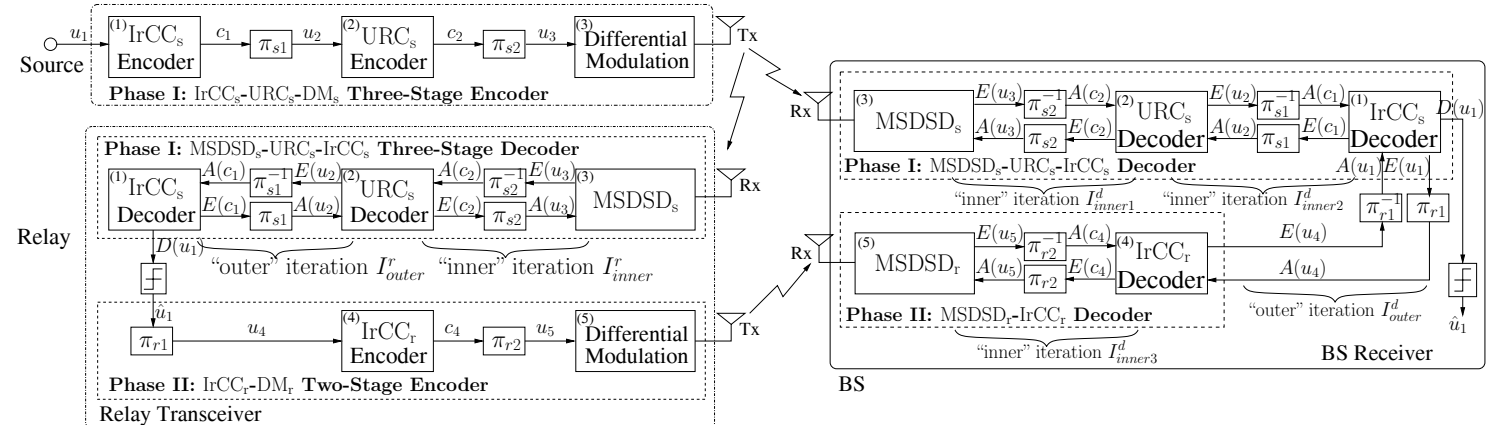

Fig. 7. Schematic of the irregular distributed differential coding encoder/decoder.

which is amalgamated with a unity-rate-code's (URC) encoder in order to create a two-stage inner code. Furthermore, an irregular convolutional code (IrCC) [23], [24] associated with an average code rate of $R_{s}$, namely $\mathrm{IrCC}_{s}$, is employed as the outer code for the sake of achieving a near-error-free transmission at an SNR close to the SR link's capacity. The IrCC proposed by Tüchler and Hagenauer, encode appropriately chosen 'fractions' of the input stream using punctured constituent convolutional codes having different code rates. The appropriate 'fractions' are determined with the aid of EXIT charts, so that the area between the EXIT curves of the inner and outer codes becomes as low as possible, which guarantees near-capacity operation, as detailed in [8]. The corresponding URC decoder assisted three-stage receiver proposed for the relay is also portrayed in Fig. 7. Specifically, at the receiver of the relay, which is constituted by three modules, namely the $\mathrm{MSDSD}_{s}$, the $\mathrm{URC}_{s}$ decoder and the $\mathrm{IrCC}_{s}$ decoder, extrinsic information is exchanged amongst the modules in a number of consecutive iterations. As shown in Fig. $7, A(\cdot)$ represents the a priori information expressed in terms of the LLRs, while $E(\cdot)$ denotes the corresponding extrinsic information. The basic idea behind the employment of the three-stage concantenated transceiver at the source MS and RS is to improve the convergence behavior of the iterative detection assisted system with the aid of the URC, thus achieving near-error-free transmission between the source and relay nodes. Essentially, since the URC employed is simply an accumulator, which has an infinite impulse response (IIR) due to its recursive encoder architecture, the resultant EXIT curve of the URC-aided inner decoder, namely the combined "MSDSD ${ }_{s}-\mathrm{URC}_{s}$ " decoder employed at the receiver of the $\mathrm{RS}$, is capable of reaching the $(1.0,1.0)$ point of the EXIT chart, as observed in Fig. 4. Furthermore, since the URC decoder employs the MAP decoding scheme, the extrinsic probability generated at the output of the URC decoder contains the same amount of information as the sequence at the input of the URC decoder [25]. In other words, the area under the inner code's EXIT curve remains the same, regardless of the URC's presence or absence. Hence, as observed in Fig. 4, a higher end-point for the EXIT curve at $I_{A}=1$ is associated with a lower starting point at $I_{A}=0$, resulting in a steeper slope for the EXIT curve, which in turn implies having a reduced error floor and a higher 'turbo-cliff' SNR, above which decoding convergence to a vanishingly low BER becomes possible, as we will demonstrate in Section VI.
At the two-stage serially concatenated transmitter of the relay of Fig. 7, the estimated data bit stream is fed through the interleaver $\pi_{r 1}$ prior to the $\mathrm{IrCC}_{r}$ encoder having an average code rate of $R_{r}$, as observed in Fig. 7, in order to construct a distributed turbo code together with the source. The distributed turbo coding scheme was first proposed in [13], where the classic turbo coding mechanism was introduced into the DF aided cooperative system in order to enhance the coding gain achieved by the repetition code constituted by the conventional relay-aided system. More Specifically, according to the principle of parallel concatenated convolutional code based turbo coding, the data and its interleaved version are encoded in parallel, using two distinct Recursive Systematic Convolutional (RSC) codes, respectively. Therefore, a distributed turbo code may be readily constructed at the relay by interleaving its received estimated source data prior to reencoding. Consequently, the proposed relay-aided cooperative system may be referred to as an Irregular Distributed Differential (IrDD) coding scheme, under the assumption of error-free decoding at the RS.

According to the principles of the distributed turbo decoding mechanism proposed in [13], the novel iterative receiver of the destination BS seen in Fig. 7 is used for decoding the IrDD coded stream jointly created by the source and relay MSs. To be specific, the first part of the iterative receiver is an amalgamated "MSDSD ${ }_{s}-\mathrm{URC}_{s}-\mathrm{IrCC}_{s}$ " iterative decoder, which is used to iteratively decode the signal directly received from the source during Phase I, while the second part is constituted of the $\mathrm{MSDSD}_{r}$ detector and the $\mathrm{IrCC}_{r}$ decoder, which is employed to iteratively decode the signal forwarded by the RS during Phase II. Note that since the $\mathrm{IrCC}_{r}$ is recursive, the EXIT curve of the combined "MSDSD ${ }_{r}-\mathrm{IrCC}_{r}$ " decoder is already capable of reaching $(1.0,1.0)$ point of the EXIT chart, thus no URC is employed at the transmitter of the RS for the sake of cost saving. Furthermore, since the "MSDSD ${ }_{s}-\mathrm{URC}_{s^{-}}$ $\mathrm{IrCC}_{s}$ " decoder and the "MSDSD ${ }_{r}-\mathrm{IrCC}_{r}$ " decoder may be regarded as the two component decoders of a turbo receiver, the extrinsic information exchange between them, which is referred to as "the "outer iteration", is expected to significantly enhance the achievable coding gain. In comparison to the conventional relay-aided cooperative system, where a simple repetition code is constructed, the extra coding gain achieved by the proposed IrDD coding scheme may be interpreted as the interleaving gain of the turbo code and the turbo processing gain of the outer iterations. 
TABLE I

TRA-OPTIMIZED NEAR-CAPACITY SYSTEM DESIGN PARAMETERS

\begin{tabular}{l|r|r}
\hline \multicolumn{2}{c}{ TRA-Optimized Near-Capacity System Design Parameters } \\
\hline \hline & $\begin{array}{r}\text { Scenario I } \\
(v=3)\end{array}$ & $\begin{array}{r}\text { Scenario II } \\
(v=2)\end{array}$ \\
\hline Fading Block Size $T_{b}$ & \multicolumn{2}{r}{0} \\
\hline Normalized Doppler Frequency $f_{d}$ & \multicolumn{2}{r}{0.5 bits/s/Hz } \\
\hline Target Bandwidth Efficiency $\eta$ & DQPSK \\
\hline Modulation & \multicolumn{2}{|c}{ MSDSD } \\
\hline Detector & 17 -subcode-based IrCC \\
\hline Channel Code & $-4.3 \mathrm{~dB}$ & $-2.1 \mathrm{~dB}$ \\
\hline Code Block Length (Source MS) & 0.56 & 0.61 \\
\hline $\begin{array}{l}\text { Theoretically Minimum Required } \gamma_{e}^{o} \\
\text { (refer to Fig. 3) }\end{array}$ & 0.52 & 0.48 \\
\hline $\begin{array}{l}\text { Optimum TRA-factor } \alpha^{o p t} \\
\text { (according to (18)) }\end{array}$ & 0.66 & 0.75 \\
\hline $\begin{array}{l}\text { Optimum Average Code Rate } R_{s} \\
\text { (according to (20)) }\end{array}$ & & \\
\hline $\begin{array}{l}\text { Optimum Average Code Rate } R_{r} \\
\text { (according to (20)) }\end{array}$ & & \\
\hline
\end{tabular}

\section{CRO NEAR-CAPACITy System DEsign}

In this section, we propose a practical framework, which enables the proposed IrDD scheme to approach the cooperative system's capacity. Both the $\operatorname{IrCC}_{s}$ and $\mathrm{IrCC}_{r}$ will be optimized based on 17 subcodes associated with the 17 dotted EXIT curves plotted in Fig. 4. Two scenarios, namely, the typical urban cellular radio Scenario I and the free-space Scenario II are considered in a time-selective block Rayleigh fading propagation environment. Without loss of generality, the target bandwidth efficiency is chosen to be $\eta=0.5$ bits/s/Hz and DQPSK modulation scheme is employed. According to Lemma 1 and Lemma 2 of Section IV-B, the optimum TRA-factor $\alpha$ and its corresponding optimum code rate pair $\left(R_{s}, R_{r}\right)$ employed by the source and RS can be obtained in the interest of maximizing the network's capacity, which are summarized in Table I together with all the other simulation parameters. As it will be shown in our forthcoming discourse, the CRO joint source-and-relay mode design procedure can be decoupled into two EXIT curve matching problems under the assumption that $a$ priori LLRs obeys a symmetric Guassian distribution, which is summarized as follows:

- Step 1: Choose a target bandwidth efficiency $\eta$;

- Step 2: Calculate the optimum TRA-factor $\alpha$ according to (18) and the corresponding optimum code rate pair $\left(R_{s}, R_{r}\right)$ employed by the source and $\mathrm{RS}$ according to (20);

- Step 3: Based on our low-complexity near-capacity design criterion relying on EXIT chart analysis, we determine the minimum number of iterations, $I_{i n n e r}^{r}$, between the $\mathrm{MSDSD}_{s}$ and the $\mathrm{URC}_{s}$ at the RS's receiver in Fig. 7, required for approaching the condition, where the increase of the area $\mathcal{A}$ under the EXIT curve of the combined "MSDSD ${ }_{s}-\mathrm{URC}_{s}$ " decoder becomes rather marginal upon further increasing $I_{i n n e r}^{r}$. We may refer to this as the state of converged information-exchange.

- Step 4: Employ the EXIT curve matching algorithm [24] to obtain the optimized weighting coefficients of the $\mathrm{IrCC}_{s}$ having an average code rate of $R_{s}$, so that a narrow but marginally open EXIT-tunnel between the EXIT curves of the inner amalgamated "MSDSD $s-\mathrm{URC}_{s}$ " de- coder and the outer $\mathrm{IrCC}_{s}$ decoder emerges at the RS's receiver.

- Step 5: Upon fixing the optimized weighting coefficients of the $\mathrm{IrCC}_{s}$ obtained in Step 4 at the source MS, determine $I_{\text {inner } 1}^{d}, I_{\text {inner } 2}^{d}$ as well as $I_{\text {inner } 3}^{d}{ }^{2}$ in a similar manner according to the low-complexity near-capacity cirterion as used in Step 3 with the aid of the EXIT chart.

- Step 6: Under the assumption of perfectly error-free DF relaying, use the EXIT curve matching algorithm of [24] to match the EXIT curve of the amalgamated "MSDSD ${ }_{r}$ $\mathrm{IrCC}_{r}$ " decoder to the target EXIT curve of the combined "MSDSD ${ }_{s}-\mathrm{URC}_{s}-\mathrm{IrCC}_{s}$ " scheme at the destination in order to obtain the optimized weighting coefficients of the $\mathrm{IrCC}_{r}$ at a fixed average code rate of $R_{r}$.

Without loss of generality, only the CRO near-capacity system design details for Scenario I of Table I are provided in the ensuing discussion, while those of Scenario II are similar, but they are omitted for the reason of space economy.

\section{A. Reduced-Complexity Near-Capacity Design at the Relay in Scenario I}

Although it is not explicitly demonstrated here owing to the lack of space, the iterative information exchange between the $\mathrm{MSDSD}_{s}$ and $\mathrm{URC}_{s}$ blocks of the relay's receiver seen in Fig. 7 approaches convergence as early as the second iteration. Hence, the number of iterations between the $\mathrm{MSDSD}_{s}$ and $\mathrm{URC}_{s}$ blocks is set to $I_{\text {inner }}^{r}=2$, when we obtain the EXIT curve of the combined "URC ${ }_{s}-\mathrm{MSDSD}_{s}$ " inner decoder of the RS, as shown in Fig. 4 and in our future simulations, in order to avoid any unnecessarily imposed complexity. In practice, for the sake of avoiding a potentially excessive complexity at the RS, while approaching the capacity, a 'higher-thannecessary' EXIT curve associated with $N_{\text {wind }}=7$ may be ensured for the combined inner "MSDSD ${ }_{s}-\mathrm{URC}_{s}$ " decoder by having a slightly 'higher-than-necessary' overall equivalent SNR, for example, $\gamma_{e}^{o}=-2.3 \mathrm{~dB}$, as depicted in Fig. 4. Then, using the EXIT curve of the amalgamated inner "MSDSD ${ }_{s}$ $\mathrm{URC}_{s}$ " decoder, the optimized weighting coefficients of the $\mathrm{IrCC}_{s}$ associated with $R_{s}=0.52$ can be obtained with the aid of the EXIT curve matching algorithm of [24], resulting in a narrow but marginally open tunnel between the EXIT curves of the amalgamated "MSDSD ${ }_{s}-\mathrm{URC}_{s}$ " decoder and the $\mathrm{IrCC}_{s}$ decoder, as seen in Fig. 4.

On the other hand, in order to further reduce the complexity imposed by the "MSDSD ${ }_{s}-\mathrm{URC}_{s}$ " decoder during the iterative decoding process at the RS, an adaptive-windowduration based scheme is proposed for the $\mathrm{MSDSD}_{s}$, where the observation window size was initially set to the smallest value of $N_{\text {wind }}=2$, which will be slightly increased, as soon as the iterative decoding process between the "MSDSD ${ }_{s}$ $\mathrm{URC}_{s}$ " decoder and the $\mathrm{IrCC}_{s}$ decoder converges. More specifically, the iterative decoding process is monitored with

\footnotetext{
${ }^{2}$ The way of determining $I_{\text {inner } 3}^{d}$ is different from that of $I_{\text {inner } 1}^{d}$, in that we commence by setting a relatively high intial value for $I_{\text {inner } 3}^{d}$ when optimizing the weighting coefficients of $\mathrm{IrCC}_{r}$. Then, we gradually reduce $I_{i n n e r 3}^{d}$ to a value, where any further reduction of $I_{i n n e r 3}^{d}$ may cause a substantial shift of the EXIT curve of the combined "MSDSD $r-\operatorname{IrCC}_{r}$ " block.
} 


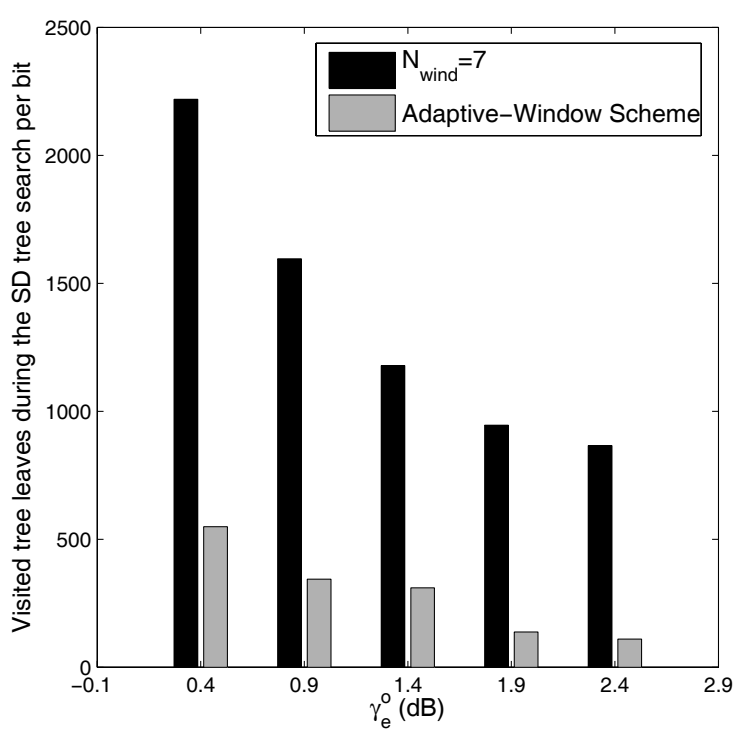

Fig. 8. Complexity reduction achieved the adaptive-window-duration scheme for the MSDSD.

the aid of the real-time MI estimation, where the MI between the transmitted coded bits having equiprobable occurrence and the extrinsic LLR may be computed without knowing the actually transmitted coded bits at the receiver as follows [26]:

$$
I=1-\frac{2}{K} \sum_{1}^{K} \frac{\log _{2}\left(1+e^{-L_{k}}\right)}{1+e^{-L_{k}}}
$$

where $K$ is the coded bit frame length. Thus, when the increase of the MI estimated at the output of the "MSDSD ${ }_{s}$ $\mathrm{URC}_{s}$ " becomes rather marginal, the value of $N_{\text {wind }}$ has to be increased to allow further benefical information exchanges among the decoders. The proposed adaptive-window-duration based scheme is characterized by Fig. 4. Indeed, the complexity imposed is significantly reduced by the adaptive-windowduration based scheme, as observed in Fig. 8, where the complexity imposed by the $\mathrm{MSDSD}_{s}$ in terms of the number of tree leaves visited during the SD tree search process per bit is plotted versus $\gamma_{e}^{o}$ for systems operating both with and without the adaptive-window-duration based scheme. Note that the complexity evaluated here for the $\mathrm{MSDSD}_{s}$ also corresponds to the number of signal vector candidates $\mathbf{s}$ enumerated during the MSDSD detection per transmitted bit. Remarkably, the complexity imposed by the $\mathrm{MSDSD}_{s}$ is substantially reduced in Fig. 8 with the aid of the adaptive-window-duration assisted scheme by as much as $75 \%$ at $\gamma_{e}^{o}=0.4 \mathrm{~dB}$, when the open EXIT tunnel created by having $N_{\text {wind }}=7$ is rather narrow. This is not unexpected, since although an increased number of iterations may be needed between the "MSDSD ${ }_{s}-\mathrm{URC}_{s}$ " decoder and the $\mathrm{IrCC}_{s}$ decoder to achieve the same amount of iteration gain, the complexity per iteration imposed by the $\operatorname{MSDSD}_{s}$ using a reduced $N_{\text {wind }}$ value is expected to be exponentially reduced when the adaptive-window-duration based scheme is employed. Thus, a potentially reduced overall complexity is imposed.

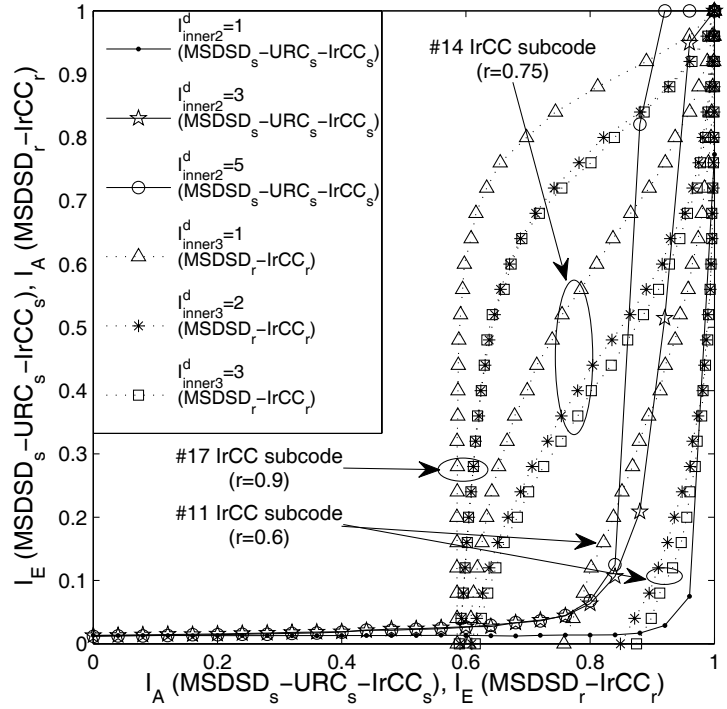

Fig. 9. EXIT curves of the amalgamated "MSDSD $-\mathrm{URC}_{s}-\mathrm{IrCC}_{s}$ " decoder and the combined "MSDSD $r-\mathrm{IrCC}_{r}$ " decoder employed by the destination BS (DQPSK, $\gamma_{e}^{o}=-2.3 \mathrm{~dB}, v=3, f_{d}=0.01$ ).

\section{B. Reduced-Complexity Near-Capacity Design at the Desti- nation in Scenario I}

In Section VI-A, the $\mathrm{IrCC}_{s}$ decoder of Fig. 7 was specifically designed to allow a near-capacity operation over the SR link with the aid of the EXIT curve matching algorithm of [24] carried out at the RS. Let us now consider the destination BS and optimize the weighting coefficients of the other $\mathrm{IrCC}$, i.e. those of the $\mathrm{IrCC}_{r}$, employed by the RS's transmitter in Fig. 7. Based on the above-mentioned desirable choice of $I_{\text {inner }}^{r}=2$, we also set the number of iterations between the $\mathrm{MSDSD}_{s}$ and $\mathrm{URC}_{s}$ blocks of the BS to $I_{\text {inner } 1}^{d}=2$. Then, we continue by determining the desirable number of iterations between the combined "MSDSD $s-\mathrm{URC}_{s}$ " decoder and the $\mathrm{IrCC}_{s}$ decoder, $I_{\text {inner } 2}^{d}$, as well as that required between the $\mathrm{MSDSD}_{r}$ and the $\operatorname{IrCC}_{r}$ arrangements, $I_{\text {inner } 3}^{d}$, by plotting the corresponding EXIT curves of the amalgamated "MSDSD ${ }_{s}-\mathrm{URC}_{s}-\mathrm{IrCC}_{s}$ " decoder as well as those of the combined "MSDSD $r-\mathrm{IrCC}_{r}$ " decoder associated with different values of $I_{\text {inner } 2}^{d}$ and $I_{\text {inner } 3}^{d}$, respectively, as seen in Fig. 9. Specifically, observe in Fig. 9 that a sharply-rising EXIT curve can be created, when using our low-complexity near-capacity design criterion, since only $I_{\text {inner } 1}^{d}=2$ iterations are required between the $\operatorname{MSDSD}_{s}$ and the $\mathrm{URC}_{s}$, while $I_{\text {inner } 2}^{d}=5$ iterations may be necessitated between the combined "MSDSD ${ }_{s}-\mathrm{URC}_{s}$ " decoder and the $\mathrm{IrCC}_{s}$ of Fig. 7. Similarly, observe the EXIT curves of the combined "MSDSD ${ }_{r}-\mathrm{IrCC}_{r}$ " decoder associated with three IrCC subcodes in Fig. 9, which indicates that although the EXIT curve of "MSDSD ${ }_{r}-\mathrm{URC}_{r}$ " can be shifted to the left by increasing the number of iterations between the $\mathrm{MSDSD}_{r}$ and the $\mathrm{IrCC}_{r}$, any further shifting of the EXIT curve to the left in the interest of creating a wider EXIT tunnel is becoming rather marginal, when the number of iterations exceeds $I_{\text {inner } 3}^{d}=2$. Hence, based on the low-complexity near-capacity design criterion, the number of iterations between the $\mathrm{MSDSD}_{r}$ and the $\mathrm{IrCC}_{r}$ blocks of Fig. 7 is chosen to be $I_{\text {inner } 3}^{d}=2$. 


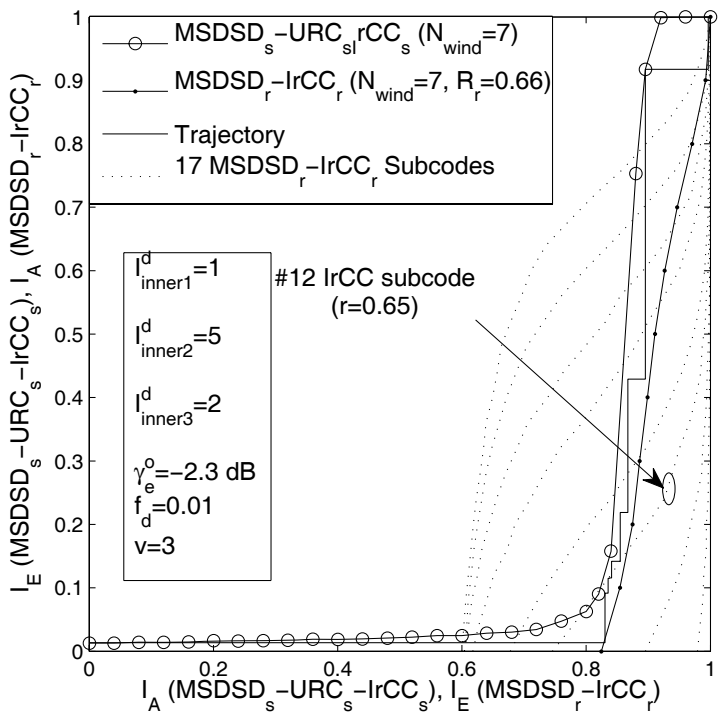

Fig. 10. Iterative decoding trajectory at the BS.

Finally, upon fixing the optimum code rate of $R_{r}=0.66$ obtained in Table I, we use the EXIT curve matching algorithm of [24] to match the SNR-dependent EXIT curve of the combined "MSDSD $r-\mathrm{IrCC}_{r}$ " decoder employed at the BS to the target EXIT curves of the amalgamated "MSDSD ${ }^{-}$$\mathrm{URC}_{s}-\mathrm{IrCC}_{s}$ " decoder of the BS, as shown in Fig. 10. Consequently, a potentially near-error-free decoding can be realized by the information exchange between the combined "MSDSD ${ }_{s}-\mathrm{URC}_{s}-\mathrm{IrCC}_{s}$ " and "MSDSD ${ }_{r}-\mathrm{IrCC}_{r}$ " decoders. However, this cannot be achieved by simply using one of 17 $\mathrm{IrCC}_{r}$ subcodes having the same code rate, as observed in Fig. 10, owing to the absence of an open EXIT tunnel.

\section{Simulation Results AND Discussions}

We have now completed the low-complexity near-capacity system design conceived for the single-relay-aided cooperative system of Fig. 1. The corresponding Monte-Carlo simulation based decoding trajectory is now plotted in Fig. 10, which reaches the $(1.0,1.0)$ point of the EXIT chart, indicating the achievement of decoding convergence to an infinitesimally low BER at near-capacity SNRs for the IrDD coding scheme proposed in Section V-B. In Fig. 11, the BER curves of a 'well-designed' cooperative systems in Scenarios I and II of Table I are portrayed in comparison to that of the conventional near-capacity point-to-point transmission based system having an identical bandwidth efficiency of $\eta=0.5 \mathrm{bits} / \mathrm{s} / \mathrm{Hz}$. Upon using the near-capacity system design of Section VI, the proposed IrDD coding scheme becomes capable of performing within about $1.8 \mathrm{~dB}$ from the corresponding single-relay-aided DDF cooperative system's DCMC capacity in both Scenarios I and II. Observe in Fig. 11 that the single-relay-aided DDF system is capable of outperforming the conventional directtransmission based system by about $2.2 \mathrm{~dB}$ for a given BER target of $10^{-5}$ in Scenario II associated with $v=2$, when both systems are designed to approach their corresponding theoretical maximum transmission rate. By contrast, in Scenario I associated with $v=3$, the single-relay-aided cooperative system becomes capable of even more significantly outperforming the

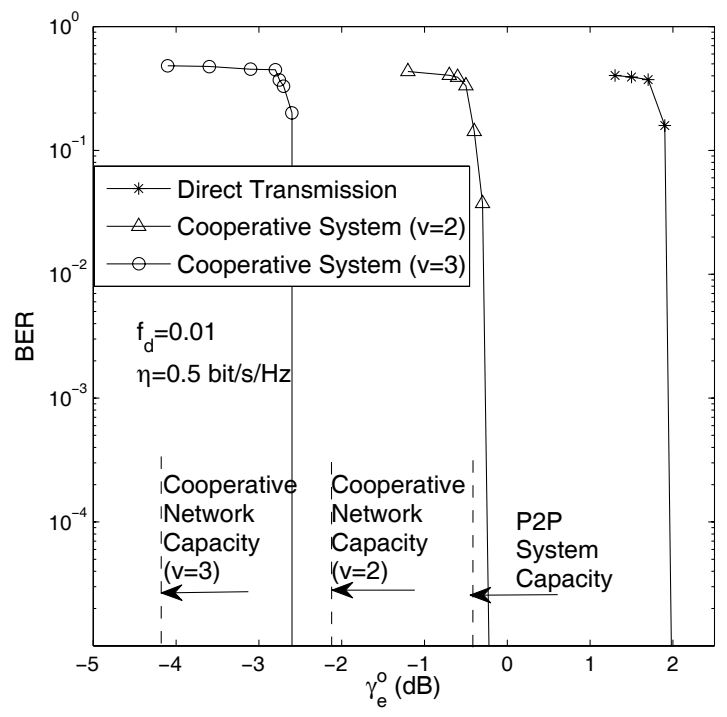

Fig. 11. Achievable BER performance of the near-capacity designed singlerelay-assisted cooperative system.

direct-transmission based system, requiring an overall transmit power, which is about $4.6 \mathrm{~dB}$ lower than that needed by the latter to achieve an infinitesimally low BER, while maintaining a bandwidth efficiency of $\eta=0.5 \mathrm{bits} / \mathrm{s} / \mathrm{Hz}$.

\section{CONCLUSION}

We proposed a CRO-based near-capacity DDF-aided cooperative system. Its DCMC capacity was investigated in comparison to that of its classic direct-transmission based counterpart in order to answer the crucial question, whether it is worth introducing cooperative mechanisms into the development of wireless networks. Based on our low-complexity nearcapacity design procedure, a novel IrDD coding scheme was conceived for DDF cooperative systems employing our lowcomplexity adaptive-window-aided SISO iterative MSDSD scheme, which operates within about $1.8 \mathrm{~dB}$ from the capacity - despite its modest complexity.

\section{REFERENCES}

[1] G. J. Foschini and M. J. Gans, "On limits of wireless communications in a fading environment when using multiple antennas," Wireless Pers. Commun., vol. 6, pp. 311-335, Mar. 1998.

[2] J. N. Laneman, D. N. C. Tse, and G. W. Wornell, "Cooperative diversity in wireless networks: efficient protocols and outage behavior," IEEE Trans. Inf. Theory, vol. 50, pp. 3062-3080, Dec. 2004.

[3] K. G. Seddik, A. K. Sadek, W. Su, and K. J. R. Liu, "Outage analysis and optimal power allocation for multinode relay networks," IEEE Signal Process. Lett., vol. 14, pp. 377-380, June 2007.

[4] T. Himsoon, W. Su, and K. J. R. Liu, "Differential transmission for amplify-and-forward cooperative communications," IEEE Signal Process. Lett., vol. 12, pp. 597-600, Sep. 2005.

[5] T. Himsoon, W. P. Siriwongpairat, W. Su, and K. J. R. Liu, "Differential modulations for multinode cooperative communications," IEEE Trans. Signal Process., vol. 56, pp. 2941-2956, July 2008.

[6] L. Wang and L. Hanzo, "The amplify-and-forward cooperative uplink using multiple-symbol differential sphere-detection," IEEE Signal Process. Lett., vol. 16, pp. 913-916, Oct. 2009.

[7] L. Wang and L. Hanzo, "The resource-optimized differentially modulated hybrid AF/DF cooperative cellular uplink using multiple-symbol differential sphere detection," IEEE Signal Process. Lett., vol. 16, pp. 965-968, Nov. 2009

[8] L. Hanzo, Y. Akhtman, L. Wang, and M. Jiang, MIMO-OFDM for LTE, WIFI and WIMAX: Coherent versus Non-Coherent and Cooperative Turbo-Transceivers. John Wiley and IEEE Press, 2010. 
[9] L. Lampe, R. Schober, V. Pauli, and C. Windpassinger, "Multiplesymbol differential sphere decoding," IEEE Trans. Commun., vol. 12, pp. 1981-1985, Dec. 2005.

[10] A. Host-Madsen and J. Zhang, "Capacity bounds and power allocation for wireless relay channel," IEEE Trans. Inf. Theory, vol. 51, pp. 20202040, June 2005.

[11] Y. Fan, C. Wang, J. Thompson, and H. V. Poor, "Recovering multiplexing loss through successive relaying using repetition coding," IEEE Trans. Wireless Commun., vol. 6, pp. 4484-4493, Dec. 2007.

[12] J. G. Proakis, Digital Communications, 4th edition. McGraw-Hill, 2000.

[13] B. Zhao and M. C. Valenti, "Distributed turbo coded diversity for relay channel," Electron. Lett., vol. 39, pp. 786-787, May 2003.

[14] M. Chiani, A. Conti, and R. Verdone, "Partial compensation signallevel-based up-link power control to extend terminal battery duration," IEEE Trans. Veh. Technol., vol. 50, pp. 1125-1131, July 2001.

[15] Y. Liang and V. V. Veeravalli, "Capacity of noncoherent time-selective Rayleigh-fading channels," IEEE Trans. Inf. Theory, vol. 50, pp. 30953110, Dec. 2004

[16] R.-R. Chen, R. Koetter, and U. Madhow, "Joint noncoherent demodulation and decoding for fast Rayleigh fading channels," in Proc. Conf. Inf. Sciences Syst., Mar. 2003.

[17] T. M. Cover and J. A. Thomas, Elements of Information Theory, 2nd edition. John Wiley \& Sons, Inc., 2006.

[18] R.-R. Chen, R. Koetter, U. Madhow, and D. Agrawal, "Joint noncoherent demodulation and decoding for the block fading channel: a practical framework for approaching Shannon capacity," IEEE Trans. Commun., vol. 51, pp. 1676-1689, Oct. 2003.

[19] H. Ochiai, P. Mitran, and V. Tarokh, "Design and analysis of collaborative diversity protocols for wireless sensor networks," in Proc. IEEE 60th Veh. Technol. Conf., 2004, vol. 7, no. 4, pp. 4645-4649.

[20] L. Wang and L. Hanzo, "Optimum time resource allocation for TDMAbased differential decode-and-forward cooperative systems: a capacity perspective," IEEE Commun. Lett., vol. 14, pp. 506-508, June 2010.

[21] V. Pauli, L. Lampe, and R. Schober, “'Turbo DPSK' using soft multiplesymbol differential sphere decoding," IEEE Trans. Inf. Theory, vol. 52, no. 4, pp. 1385-1398, 2006.

[22] A. Ashikhmin, G. Kramer, and S. ten Brink, "Extrinsic information transfer functions: model and erasure channel properties," IEEE Trans. Inf. Theory, vol. 50, pp. 2657-2673, Nov. 2004.

[23] M. Tüchler and J. Hagenauer, "EXIT charts of irregular codes," in Proc. Conf. Inform. Science Syst. (CDROM), 2002, pp. 20-22.

[24] M. Tüchler, "Design of serially concatenated systems depending on the block length," IEEE Trans. Commun., vol. 52, pp. 209-218, Feb. 2004.

[25] J. Kliewer, A. Huebner, and D. J. Costello, "On the achievable extrinsic information of inner decoders in serial concatenation," in Proc. International Symp. Inf. Theory, July 2006, pp. 2680-2684.

[26] S. Ibi, T. Matsumoto, R. Thoma, S. Sampei, and N. Morinaga, "EXIT chart-aided adaptive coding for multilevel BICM with turbo equalization in frequency-selective MIMO channels," IEEE Trans. Veh. Technol., vol. 56, pp. 3757-3769, Nov. 2007.

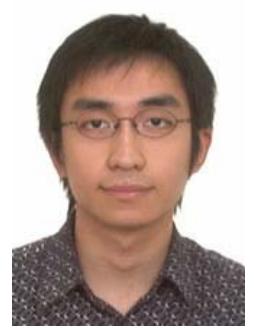

Li Wang (S'09-M'10) was born in Chengdu, China, in 1982. He received his B.Eng. degree in information engineering from Chengdu University of Technology (CDUT), Chengdu, China, in 2005 and his M.Sc. degree with distinction in radio frequency communication systems from the University of Southampton, UK, in 2006. Between October 2006 and January 2010, he was a Ph.D. student in the Communications Group, School of Electronics and Computer Science, University of Southampton, and participated in the Delivery Efficiency Core Research Programme of the Virtual Centre of Excellence in Mobile and Personal Communications (Mobile VCE). He was awarded a joint EPSRC and Mobile VCE full scholarship. Upon the completion of his Ph.D. in January 2010, he conducted research as a senior research fellow in the School of Electronics and Computer Science at the University of Southampton. Currently, he is involved in Project \#7 of the Indian-UK Advanced Technology Centre (IU-ATC): Advanced air interface technique for MIMO-OFDM and cooperative communications. His research interests include space-time processing, channel coding, multi-user detection, non-coherent transmission techniques, iterative detection, EXIT-chart-aided system design, MIMO-OFDM, and cooperative systems.

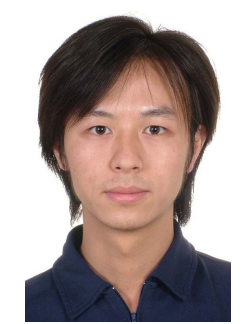

Lingkun Kong received the B.Eng. degree in information engineering and the M.Eng. degree in communications and signal processing from Southeast University, Nanjing, China, in 2004 and 2006, respectively. He received the Ph.D. degree in wireless communications from the University of Southampton, UK, in 2010. His research interests include channel coding, space-time coding, and iterative detection techniques, as well as co-located and distributed MIMO systems.

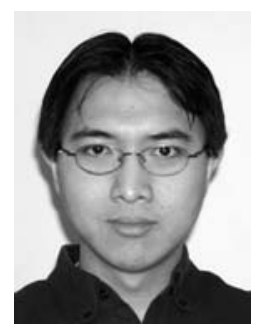

Soon Xin Ng (S'99-M'03-SM'08) received the B.Eng. degree (First Class) in electronics engineering and the Ph.D. degree in wireless communications from the University of Southampton, Southampton, U.K., in 1999 and 2002, respectively. From 2003 to 2006, he was a postdoctoral research fellow working on collaborative European research projects known as SCOUT, NEWCOM, and PHOENIX. Since August 2006, he has been a member of the academic staff in the School of Electronics and Computer Science, University of Southampton. He is involved in the OPTIMIX European project, as well as the IU-ATC and UC4G projects. His research interests include adaptive coded modulation, coded modulation, channel coding, space-time coding, joint source and channel coding, iterative detection, OFDM, MIMO, cooperative communications, and distributed coding. He has published over 120 papers and co-authored two John Wiley/IEEE Press books in this field. He is a senior member of the IEEE and a fellow of the Higher Education Academy in the UK.

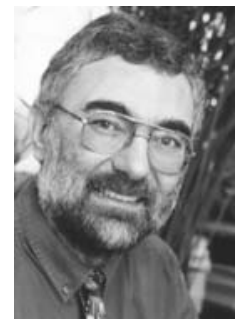

Lajos Hanzo FREng, FIEEE, FIET, DSc, received his degree in electronics in 1976 and his doctorate in 1983. In 2009, he was awarded the honorary doctorate "Doctor Honaris Causa" by the Technical University of Budapest. During his 35-year career in telecommunications, he has held various research and academic posts in Hungary, Germany, and the UK. Since 1986, he has been with the School of Electronics and Computer Science, University of Southampton, UK, where he holds the chair in telecommunications. He has co-authored 20 John Wiley/IEEE Press books on mobile radio communications totalling in excess of 10,000 pages, published about 990 research entries at IEEE Xplore, acted as TPC Chair of IEEE conferences, presented keynote lectures, and been awarded a number of distinctions. Currently, he is directing an academic research team working on a range of research projects in the field of wireless multimedia communications sponsored by industry, the Engineering and Physical Sciences Research Council (EPSRC) UK, the European IST Programme, and the Mobile Virtual Centre of Excellence (VCE), UK. He is an enthusiastic supporter of industry and academic liaison, and offers a range of industrial courses. He is also a Governor of the IEEE VTS. Since 2008, he has been the Editor-in-Chief of the IEEE Press, and since 2009, a Chaired Professor at Tsinghua University, Beijing. For further information on research in progress and associated publications, please refer to http://wwwmobile.ecs.soton.ac.uk. 